\title{
Infrared-Activated Proton Transfer in Aqueous Nafion Proton-Exchange-Membrane Nanochannels
}

\begin{abstract}
Liyuan Liu and Huib J. Bakker
FOM Institute AMOLF, Science Park 104, 1098 XG Amsterdam, The Netherlands (Received 25 September 2013; revised manuscript received 19 March 2014; published 26 June 2014)

We report on the observation of a strong reorganization of the proton hydration structure in hydrated Nafion membranes following single-quantum excitation of a proton vibration with $\sim 4 \mu \mathrm{m}$ light pulses. The reorganization takes place with a time constant of $170 \pm 20 \mathrm{fs}$ and leads to a strong red shift of the excited proton vibration and the rise of new waterlike $\mathrm{O}-\mathrm{H}$ stretch absorption bands. These observations can be explained from a vibrational-excitation-induced change of the proton-hydration structure that involves transfer of the proton charge. The results are consistent with recent quantum molecular dynamics simulations of proton transfer in Nafion membranes.
\end{abstract}

DOI: 10.1103/PhysRevLett.112.258301

PACS numbers: 82.47.Gh, 78.47.J-, 82.50.Bc, 82.53.Xa

Light-induced chemical reactions are extremely widespread and important. Examples are photosynthesis and the formation of ozone. In most cases, the reaction is induced by excitation of an electronic degree of freedom of the molecule that leads to a weakening of one or more chemical bonds. The triggering of a chemical reaction by excitation of a molecular vibration is much less common and usually requires multiquantum excitation to a highly excited vibrational state [1-3].

Single-quantum vibrational excitation is usually not sufficient to trigger a chemical reaction. However, for some reactive systems, single-quantum vibrational excitation can increase the rate of a particular reaction channel, as has been demonstrated for bimolecular reactions between small molecules and radicals in the gas phase [4,5]. In the condensed phase, the triggering of a chemical reaction by vibrational excitation is more complicated, as the intermolecular interactions can lead to dissipation of the energy of the excited vibration before the reaction can take place. Up to now, the only example of such a condensed-phase reaction is the cis-trans isomerization of nitrous acid (HONO) following excitation of the $\mathrm{O}-\mathrm{H}$ stretch vibration [6,7]. For this infrared-driven (IR-driven) reaction to occur, the molecule has to be embedded in a rare-gas matrix at low temperatures to limit the intermolecular interactions that would lead to dissipation.

Intermolecular interactions can also have a positive effect in enabling a light-driven chemical reaction. The groups of Ando and Hynes $[8,9]$ predicted that the strong coupling between an excited vibration and surrounding water molecules can lead to proton transfer. They calculated that the excitation of the $\mathrm{H}-\mathrm{F}$ vibration of $\mathrm{HF}$ in water would lead to transfer of a proton from $\mathrm{HF}$ to a neighboring water molecule. Up to now this type of vibrational-excitationinduced proton transfer has eluded experimental observation.

Here we find evidence for the activation of proton transfer by single-quantum vibrational excitation in the nanochannels of a hydrated Nafion membrane at room temperature. Nafion is a sulfonated tetrafluoroethelyne (Teflon)-based fluoropolymer and forms the most widely used proton exchange membrane in hydrogen fuel cells [10]. Nafion possesses hydrophilic sulfonated perfluorovinyl side chains that form nanoscaled hydrophilic domains. The counter cations of the negatively charged sulfonate groups can be formed by protons, and in case the Nafion is hydrated, meaning that the membrane channels contain water molecules, the protons become mobile [11]. The introduction of protons leads to a broadband absorption between 1500 and $3100 \mathrm{~cm}^{-1}$ (see the Supplemental Material [12], which includes Refs [13,14]). Recent quantum molecular dynamics simulations of Nafion employing the self-consistent multistate empirical valence bond method showed that the hydrated proton in Nafion acquires similar proton hydration structures as in liquid water; i.e., the proton can be hydrated in an Eigenlike structure or in a Zundellike structure [15-17]. The Eigen hydration structure is formed by an $\mathrm{H}_{3} \mathrm{O}^{+}$ion of which the three $\mathrm{O}-\mathrm{H}$ groups are strongly hydrogen bonded to three neighboring oxygen atoms, thus forming an $\mathrm{H}_{9} \mathrm{O}_{4}^{+}$structure. In the Zundel structure, the proton is flanked by two water molecules, thus forming an $\mathrm{H}_{5} \mathrm{O}_{2}^{+}$structure. The quantum molecular dynamics simulations show that the proton hydration structure is Zundellike in the first hydration shell of the sulfonate counterion and Eigenlike at larger distances from this ion $[15,16]$.

We study the properties of water molecules and protons in hydrated nafion nanochannels using polarization-resolved femtosecond vibrational spectroscopy employing independently tunable excitation and detection pulses. The central frequency $\nu_{\text {exc }}$ of the excitation pulse is tuned from 2850 to $3700 \mathrm{~cm}^{-1}$, and the detection frequency $\nu_{\text {det }}$ is tuned from 1500 to $3700 \mathrm{~cm}^{-1}$. We use a relatively narrow-band excitation pulse (with a bandwidth of $\sim 110 \mathrm{~cm}^{-1}$ ) to ensure the selective excitation of molecular vibrations of water 
molecules or particular proton hydration structures. In the experiment, we monitor the excitation-induced absorption changes $\Delta \alpha$ and the anisotropic $R$ of $\Delta \alpha$ as a function of the central excitation frequency, the detection frequency, and the time delay between the excitation and the detection pulses.

Figure 1 shows two-dimensional infrared (2D-IR) spectra of hydrated Nafion nanochannels for four different time delays between the excitation and the detection pulses. For $\nu_{\text {exc }}=3500 \mathrm{~cm}^{-1}, \nu_{\text {det }}=3500 \mathrm{~cm}^{-1}$ we observe a bleaching signal of the fundamental $v=0 \rightarrow 1$ absorption of the water $\mathrm{O}-\mathrm{H}$ stretch vibrations. The induced absorption signal observed for $\nu_{\mathrm{exc}}=3500 \mathrm{~cm}^{-1}, \nu_{\mathrm{det}}=3300 \mathrm{~cm}^{-1}$ results from the $v=1 \rightarrow 2$ excited-state absorption of the water $\mathrm{O}-\mathrm{H}$ stretch vibrations. The $2 \mathrm{D}$-spectra at different time delays illustrate that the excited water $\mathrm{O}-\mathrm{H}$ stretch vibrations decay relatively slowly, much slower than the $\mathrm{O}-\mathrm{H}$ stretch vibrations of bulk $\mathrm{H}_{2} \mathrm{O}$ for which $T_{1}$ is $\sim 250$ fs [18]. This finding agrees with the results of a study of the vibrational relaxation of isotopically diluted water in nafion membranes [19].
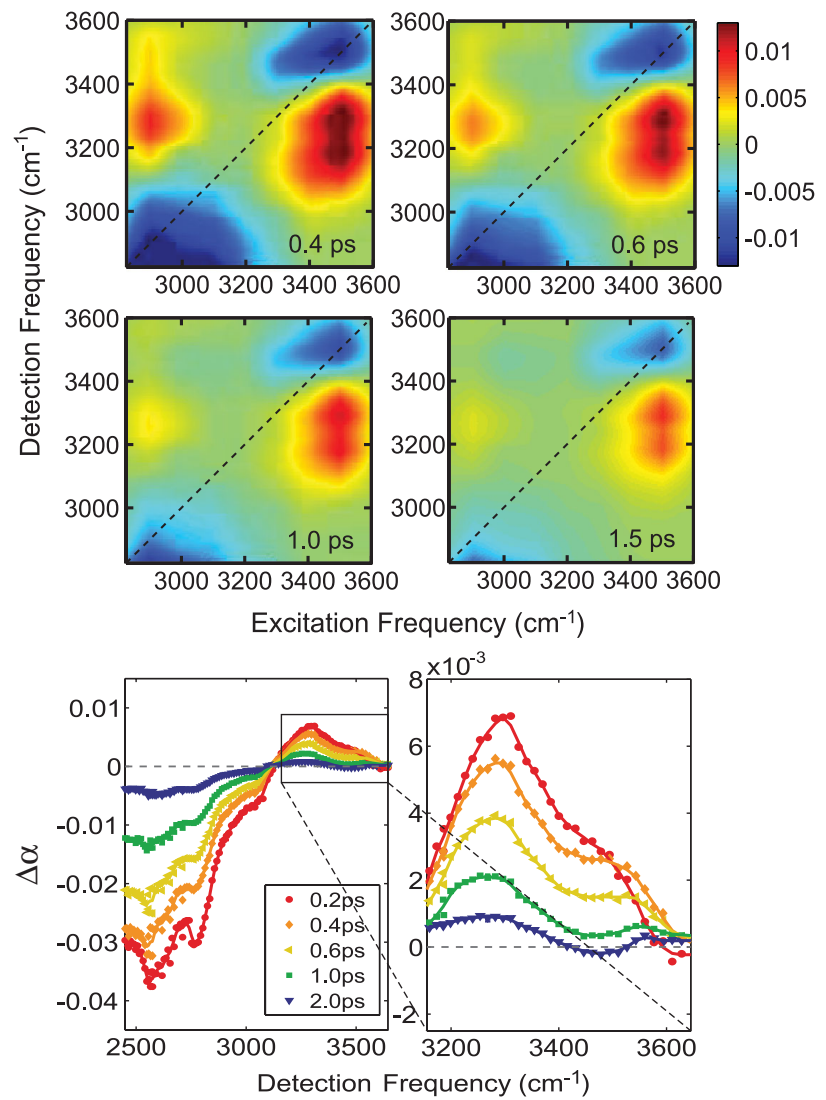

FIG. 1 (color). (Upper panel) Isotropic 2D-IR spectra of a Nafion membrane at a hydration level of three water molecules per sulfonate group. The spectra show strong diagonal signals at the water vibrations $\left(\sim 3500 \mathrm{~cm}^{-1}\right)$ and the Eigen proton vibrations $\left(\sim 2900 \mathrm{~cm}^{-1}\right)$, and cross-peak signals between the proton and the water vibrations. (Lower panel) Cuts at $\nu_{\mathrm{exc}}=2900 \mathrm{~cm}^{-1}$ of 2D-IR spectra at different delay times. The induced absorption bands at 3270 and $3520 \mathrm{~cm}^{-1}$ are also shown enlarged.
For $\quad \nu_{\text {exc }}=2900 \mathrm{~cm}^{-1}, \quad \nu_{\text {det }}=2900 \mathrm{~cm}^{-1}$ a broad bleaching signal is observed. We assign this bleaching signal to the excitation of an $\mathrm{O}-\mathrm{H}$ vibration of the hydronium $\mathrm{H}_{3} \mathrm{O}^{+}$core of an Eigen proton hydration structure [13,20-22]. The Eigen structures are dynamically distorted, thus leading to a very broad response around $2600 \mathrm{~cm}^{-1}$. The 2D spectra also show clear cross-peak signals: the excitation of the proton vibration with $\nu_{\mathrm{exc}}=2900 \mathrm{~cm}^{-1}$ leads to a signal at $\nu_{\text {det }}>3250 \mathrm{~cm}^{-1}$. By taking a cut of the 2D spectrum at a particular excitation frequency, we obtain a transient spectrum. The lower panel of Fig. 1 shows transient spectra at five different delay times for $\nu_{\text {exc }}=2900 \mathrm{~cm}^{-1}$. The transient spectra show a bleaching signal for $\nu_{\text {det }}$ between 2500 and $3100 \mathrm{~cm}^{-1}$ and induced absorption signals at $\nu_{\text {det }}=3270 \mathrm{~cm}^{-1}$ and $\nu_{\text {det }}=3520 \mathrm{~cm}^{-1}$. These latter signals constitute the cross-peak signal in the 2D-IR spectra shown in the upper panel of Fig. 1.

For a complex molecular system like hydrated Nafion, the observation of cross-peaks can have different origins. One of these origins is vibrational coupling, implying that the excitation of a vibration of the system induces a change in frequency of other nearby vibrations. In this case, the cross-peak signal should have the same dynamics as the excited vibration [23]. However, in the left panel of Fig. 2, it is seen that the induced absorption signal at $\nu_{\text {det }}=$ $3270 \mathrm{~cm}^{-1}$ rises with a delay of $\sim 200 \mathrm{fs}$ with respect to the excitation, thus ruling out vibrational coupling as its origin.

Another potential origin of the cross-peak signal is a heating effect. The energy of the excited proton vibration is eventually converted to heat which can lead to a breaking of hydrogen bonds and a blueshift of the proton vibration to frequencies $>3000 \mathrm{~cm}^{-1}$. To test this explanation, we measured the anisotropy of the vibrational signals following excitation of the proton vibration (right panel of Fig. 2). The anisotropy at $\nu_{\text {det }}=2900 \mathrm{~cm}^{-1}$ is initially positive $(\sim 0.3)$, while the anisotropy at $\nu_{\text {det }}=3270 \mathrm{~cm}^{-1}$ is initially
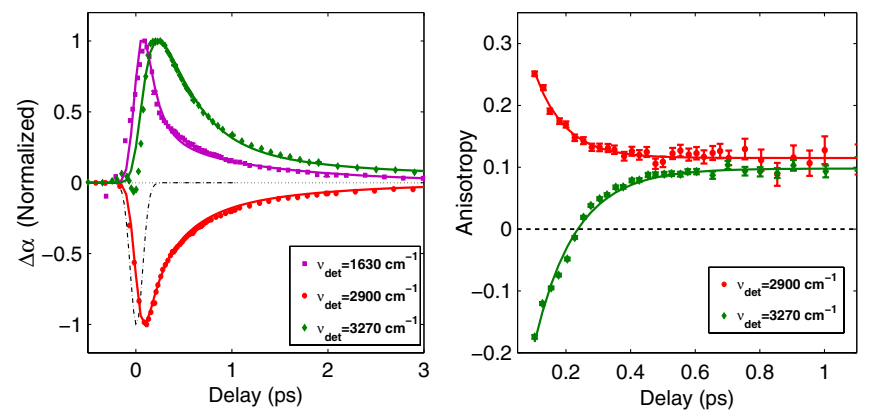

FIG. 2 (color online). (Left panel) Normalized isotropic absorption changes as a function of delay at three different detection frequencies after excitation with $\nu_{\text {exc }}=2900 \mathrm{~cm}^{-1}$. A positive signal implies an induced absorption, a negative signal a bleaching signal. The solid lines result from a fit to a four-level kinetic model (described in the Supplemental Material [12]). (Right panel) Anisotropy measured at two detection frequencies after excitation with $\nu_{\text {exc }}=2900 \mathrm{~cm}^{-1}$. 
negative $(\sim-0.1)$. The induced absorption band at $\nu_{\text {det }}=$ $3520 \mathrm{~cm}^{-1}$ shows the same initial negative anisotropy (not shown). The initial negative anisotropy of the bands at $3270 \mathrm{~cm}^{-1}$ and $3520 \mathrm{~cm}^{-1}$ indicates that the associated vibrational transition dipole moments are at a relatively large angle with respect to that of the excited vibration. The observation of an initial negative anisotropy rules out the possibility that the induced absorption bands would be generated by a heating effect because in this case, the induced absorption bands would have zero anisotropy (complete thermalization), or a positive anisotropy (in case the heating would mainly affect the originally excited vibration).

Cross-peak signals can also be caused by structural relaxation and equilibration processes that lead to a change of the character and frequencies of the excited and nonexcited vibrations [24]. We observe that the rise of the cross peaks is accompanied by a strong red shift of the bleaching signal of the excited proton vibration (top panel of Fig. 3). This red shift points at a shortening and strengthening of the hydrogen bond donated by the excited $\mathrm{O}-\mathrm{H}$ stretch vibration [25], implying a strong structural relaxation. We find that the bleaching shifts much further than to the center frequency of $2600 \mathrm{~cm}^{-1}$ of the absorption spectrum of the Eigen proton vibration [20-22]. The strong red shift of the bleaching indicates that the excited $v=1$ state of the Eigen vibration evolves into an excited $v=1$ state of an $\mathrm{O} \cdots \mathrm{H} \cdots \mathrm{O}$ Zundel vibration. This vibration has a frequency of $\sim 1100 \mathrm{~cm}^{-1}$, much lower than the Eigen vibration [20-22].

The rise of the cross peaks at $\nu_{\text {det }}=3270 \mathrm{~cm}^{-1}$ and $\nu_{\text {det }}=3520 \mathrm{~cm}^{-1}$ can be well explained from the excitation-induced structural relaxation from Eigen to Zundel. When the excited $\mathrm{O}-\mathrm{H}$ vibration in hydronium core of Eigen complex evolves to a Zundel proton vibration, the two other not excited $\mathrm{O}-\mathrm{H}$ modes of the Eigen complex will turn into higher frequency $\mathrm{O}-\mathrm{H}$ stretch vibrations of water molecules flanking the Zundel proton. The frequencies of the $\mathrm{O}-\mathrm{H}$ stretch vibrations of water molecules flanking a proton in an $\mathrm{H}_{5} \mathrm{O}_{2}^{+}$Zundel hydration structure indeed correspond well to the frequencies of $3270 \mathrm{~cm}^{-1}$ and $3520 \mathrm{~cm}^{-1}$ of the cross peaks [20,21]. Furthermore, the delay time at which the induced absorption bands of the Zundel proton-hydration structure reach their maximum (Fig. 2) is similar to the delay time at which the bleaching below $2200 \mathrm{~cm}^{-1}$ reaches its maximum (top panel of Fig. 3). Finally, these vibrations are at a quite large angle with respect to the proton vibration, which agrees with the initial negative value of the anisotropy of the cross-peak signals.

In the middle panel of Fig. 3, we present a schematic picture of the evolution from Eigen to Zundel and the corresponding changes in vibrational frequencies. These structures are based on the results of quantum molecular dynamics simulations of Nafion [15-17].
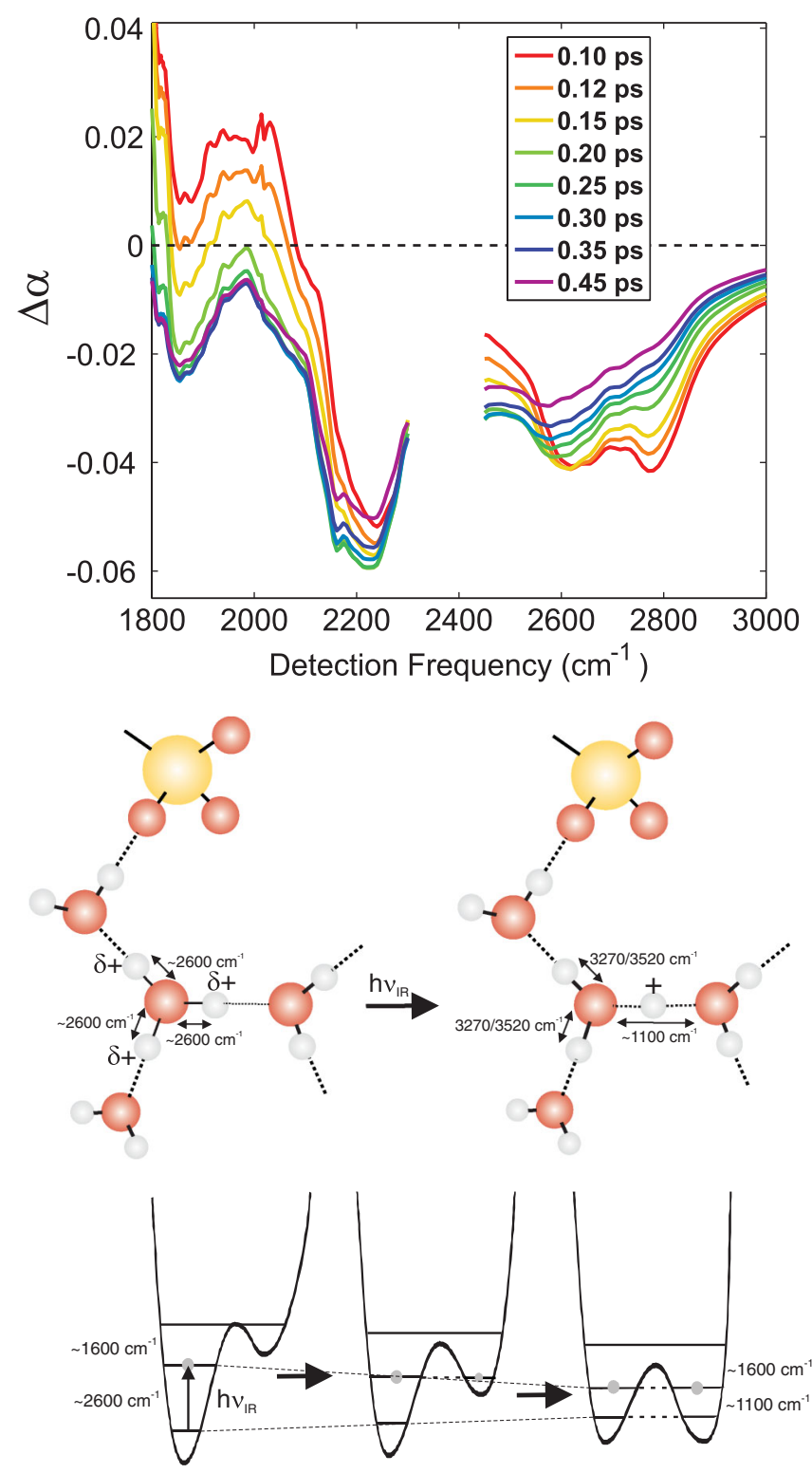

FIG. 3 (color). (Top panel) Isotropic transient absorption spectra of a proton-terminated Nafion membrane at different delay times at detection frequencies $\nu_{\text {det }}$ ranging from 1800 to $3000 \mathrm{~cm}^{-1}$. The hydration level amounts to approximately three water molecules per sulfonate group. The Nafion membrane is excited with $\nu_{\mathrm{exc}}=2900 \mathrm{~cm}^{-1}$. The spectral region between 2300 and $2450 \mathrm{~cm}^{-1}$ is missing because of the strong absorption of Nafion in this frequency region. (Middle panel) Schematic picture of the evolution from an Eigen proton hydration structure to a Zundel hydration structure following excitation of one of the proton vibrations of the Eigen structure. The vibrational frequencies of the different modes of the hydration structures are indicated. (Lower panel) The corresponding adiabatic change in the shape of the vibrational potential of the proton.

Quantum molecular dynamics simulations showed that proton transfer in liquid water involves a special pair dance in which the central hydronium ion of the Eigen structure can form a special pair with one of the three surrounding 
water molecules [26]. For this special pair, the hydrogen bond is shorter than for the other two $\mathrm{O}-\mathrm{H}$ groups of the hydronium ion. Our observations indicate that the excitation of an $\mathrm{O}-\mathrm{H}$ stretch vibration of the $\mathrm{H}_{3} \mathrm{O}^{+}$core of an Eigenlike cation leads to a contraction of the hydrogen bond at this excited $\mathrm{O}-\mathrm{H}$ group. As a result, the excited $\mathrm{O}-\mathrm{H}$ group and the water molecule to which this group is hydrogen bonded become the special pair. The driving force for contraction of the hydrogen bond is the lowering of the energy of the excited state of the proton vibration. Upon contraction of the hydrogen bond, the vibrational potential changes from a strongly asymmetric and deep potential to a symmetric and broad potential, as illustrated in the lower panel of Fig. 3. The energy of the first excited vibrational state is much lower in the symmetric potential than in the original asymmetric potential. The change of the vibrational potential is accompanied by a transfer of proton charge from the nonexcited $\mathrm{O}-\mathrm{H}$ groups of the original $\mathrm{H}_{3} \mathrm{O}^{+}$ion to the vibrationally excited Zundel proton. This scenario agrees with the mechanism of infrared activated adiabatic proton transfer proposed by Hynes and others [8,9]. After relaxation of the $v=1$ state in the Zundel potential, the system will evolve back to an Eigen hydration structure that can be the same as the one before the excitation or that can be translated by one water molecule, thus completing a successful proton-transfer reaction.

To determine the time constants of the transient spectral changes we fitted the data shown in the left panel of Fig. 2 to a kinetic model (see the Supplemental Material [12]). The fit results are represented by the solid curves in Fig. 2. The bleaching signal at $\nu_{\text {det }}=2900 \mathrm{~cm}^{-1}$ and the induced absorption at $\nu_{\text {det }}=1630 \mathrm{~cm}^{-1}$ show a pulse-width limited rise (Fig. 2), which indicates that these signals are both due to the direct excitation of the Eigen proton vibration. The excitation of the $v=1$ state of the Eigen vibration not only leads to a bleaching of the fundamental $v=0 \rightarrow 1$ transition but also to a new absorption band starting from the $v=1$ state corresponding to the $v=1 \rightarrow 2$ transition. This latter transition is very broad and at a much lower frequency than the fundamental transition as a result of the anharmonicity of the proton vibration. We thus assign the broadband absorption at early delays at frequencies $\nu_{\text {det }}<$ $1850 \mathrm{~cm}^{-1}$ (Fig. 3) to the $v=1 \rightarrow 2$ excited state absorption of the Eigen proton vibration, which implies that this proton vibration possesses a very large anharmonicity. This assignment agrees with previous calculations of the vibrational levels of the hydrogen-bonded $\mathrm{O}-\mathrm{H} \cdots \mathrm{O}$ system based on the Lippincott-Schröder potential [27,28].

From the fit, we find that the Eigen-Zundel transfer and the vibrational relaxation have time constants of $170 \pm$ $20 \mathrm{fs}$ and $350 \pm 30 \mathrm{fs}$, respectively. The Eigen-Zundel transfer leads to a decay of the stimulated emission contribution to the bleaching signal at $2900 \mathrm{~cm}^{-1}$ ( $\sim 50 \%$ of the total bleaching signal), a decay of the $v=$ $1 \rightarrow 2$ excited state absorption of the Eigen proton vibration at $1630 \mathrm{~cm}^{-1}$, a rise of a bleaching signal at frequencies $<2300 \mathrm{~cm}^{-1}$, and a rise of the induced absorption signals at 3270 and $3520 \mathrm{~cm}^{-1}$. The vibrational relaxation leads to a decay of the induced absorptions at 3270 and $3520 \mathrm{~cm}^{-1}$ and to a further decay of the bleaching signal of the Eigen proton vibration (repletion of the ground state). The vibrational relaxation eventually results in a local heating effect that is associated with a small residual change in absorption. This absorption change decays by transfer of thermal energy to the Nafion backbone. We model this final equilibration process as an exponential decay process with a time constant of $1.3 \pm 0.2 \mathrm{ps}$.

The anisotropy of the excited proton vibration at $\nu_{\text {det }}=$ $2900 \mathrm{~cm}^{-1}$ and the induced absorption at $\nu_{\text {det }}=$ $3270 \mathrm{~cm}^{-1}$ both relax to a value of $\sim 0.1$ with a time constant of $120 \pm 30 \mathrm{fs}$ (Fig. 2). This final value of the anisotropy indicates that the excitation has randomized in the plane of the central $\mathrm{H}_{3} \mathrm{O}^{+}$ion of the Eigen hydration structure [29], meaning that the vibrational excitation is delocalized over the three $\mathrm{O}-\mathrm{H}$ groups of the central $\mathrm{H}_{3} \mathrm{O}^{+}$ ion. The hydrogen bonds between the central hydronium ion and the three neighboring water molecules are constantly fluctuating in length and strength. These fluctuations modulate the frequencies of the three $\mathrm{O}-\mathrm{H}$ vibrations of the central hydronium ion, thereby enabling rapid energy transfer between these vibrations. After the vibrational excitation has become delocalized over the three $\mathrm{O}-\mathrm{H}$ vibrations, the excitation-induced formation of the special pair can occur at each of the three $\mathrm{O}-\mathrm{H}$ groups. As a result, the anisotropy of both the bleaching and the induced absorption signals become equal to 0.1 . Only directly after the excitation there is a preference for Eigen-Zundel transfer along the $\mathrm{O}-\mathrm{H}$ group that was initially excited, leading to the initial negative anisotropy of the induced absorption at $\nu_{\text {det }}=3270 \mathrm{~cm}^{-1}$ and $\nu_{\text {det }}=3520 \mathrm{~cm}^{-1}$.

For bulk liquid water, the excitation of the proton vibration of the Eigen structure does not lead to proton transfer, probably because the vibrational lifetime $T_{1}$ of the proton vibration in bulk water is only $110 \pm 20 \mathrm{fs}$ [30]. In the Nafion nanochannels, proton transfer can occur within the lifetime because the proton vibration has a relatively long $T_{1}$ of $350 \pm 30 \mathrm{fs}$. This favorable comparison of the time scales of proton transfer and vibrational relaxation finds its origin in the molecular structure of the hydrated Nafion nanochannel. Recent quantum molecular dynamics simulations showed that in Nafion nanochannels the proton diffusion via Grotthuss conduction is anticorrelated to the diffusion via thermal motions of the proton hydration structures (vehicular diffusion) [15-17], as a result of the strong association of the hydrated protons with the sulfonate counter ions and the reduced dimensionality of the water network in which the diffusion takes place. The strong association with sulfonate can be the origin of the relatively slow vibrational relaxation of the excited proton vibration. A similar slowing down has been observed for 
the hydroxyl vibrations of water molecules bound to sulfonate [31]. The limited extension of the water network in the Nafion nanochannel may also play a role. This network can show a strong intermolecular coupling in one direction, thereby enabling excitation induced proton transfer, while showing little intermolecular coupling in other directions, thus making the vibrational relaxation relatively slow.

This work is part of the research program of the "Stichting voor Fundamenteel Onderzoek der Materie (FOM)," which is financially supported by the "Nederlandse organisatie voor Wetenschappelijk Onderzoek (NWO)." The authors thank Ellen Backus and Hinco Schoenmaker for technical support.

*Corresponding author. bakker@amolf.nl

[1] N. F. Scherer and A. H. Zewail, J. Chem. Phys. 87, 97 (1987).

[2] Y. Kim, T. Komeda, and M. Kawai, Phys. Rev. Lett. 89, 126104 (2002).

[3] J. I. Pascual, N. Lorente, Z. Song, H. Conrad, and H. P. Rust, Nature (London) 423, 525 (2003).

[4] J. P. Camden, H. A. Bechtel, D. J. Brown, and R. N. Zare, J. Chem. Phys. 123, 134301 (2005).

[5] S. Yoon, R. J. Holiday, and F. F. Crim, J. Phys. Chem. B 109, 8388 (2005).

[6] R. T. Hall and G. C. Pimentel, J. Chem. Phys. 38, 1889 (1963).

[7] R. Schanz, V. Botan, and P. Hamm, J. Chem. Phys. 122, 044509 (2005).

[8] K. Ando and J. T. Hynes, J. Phys. Chem. A 103, 10398 (1999).

[9] T. Joutsuka and K. Ando, J. Phys. Chem. A 115, 671 (2011).

[10] K. A. Mauritz and R. B. Moore, Chem. Rev. 104, 4535 (2004).

[11] A. Anantaraman and C. Gardner, J. Electroanal. Chem. 414, 115 (1996).
[12] See Supplemental Material at http://link.aps.org/ supplemental/10.1103/PhysRevLett.112.258301 for additional and supporting experimental results and description of the kinetic model.

[13] M. Laporta, M. Pegoraroa, and L. Zanderighi, Phys. Chem. Chem. Phys. 1, 4619 (1999).

[14] V. Glezakou, M. Dupuis, and C. J. Mundy, Phys. Chem. Chem. Phys. 9, 5752 (2007).

[15] M. K. Petersen and G. A. Voth, J. Phys. Chem. B 110, 18594 (2006).

[16] S. Feng and G. A. Voth, J. Phys. Chem. B 115, 5903 (2011).

[17] Y.-L. S. Tse, A. M. Herring, K. Kim, and G. A. Voth, J. Phys. Chem. C 117, 8079 (2013).

[18] A. J. Lock and H. J. Bakker, J. Chem. Phys. 117, 1708 (2002).

[19] D. E. Moilanen, I. R. Piletic, and M. D. Fayer, J. Phys. Chem. C 111, 8884 (2007).

[20] J. Kim, U. W. Schmitt, J. A. Gruetzmacher, G. A. Voth, and N. E. Scherer J. Chem. Phys. 116, 737 (2002).

[21] J. M. Headrick et al., Science 308, 1765 (2005).

[22] J. Xu, Y. Zhang, and G. A. Voth, J. Phys. Chem. Lett. 2, 81 (2011).

[23] S. Woutersen and P. Hamm, J. Phys. Chem. B 104, 11316 (2000).

[24] J. R. Zheng, K. W. Kwak, J. Asbury, X. Chen, I. R. Piletic, and M. D. Fayer, Science 309, 1338 (2005).

[25] W. Mikenda, J. Mol. Struct. 147, 1 (1986).

[26] O. Markovitch, H. Chen, S. Izvekov, F. Paesani, G. A. Voth, and N. Agmon, J. Phys. Chem. B 112, 9456 (2008).

[27] H. J. Bakker, H.-K. Nienhuys, G. Gallot, N. Lascoux, G. M. Gale, J.-C. Leicknam, and S. Bratos, J. Chem. Phys. 116, 2592 (2002).

[28] F. Perakis, S. Widmer, and P. Hamm, J. Chem. Phys. 134, 204505 (2011).

[29] J. R. Lakowicz, Principles of Fluorescence Spectroscopy (Springer, New York, 2006), p. 421.

[30] S. Woutersen and H. J. Bakker, Phys. Rev. Lett. 96, 138305 (2006).

[31] I. R. Piletic, D. E. Moilanen, D. B. Spry, N. E. Levinger, and M. D. Fayer, J. Phys. Chem. A 110, 4985 (2006). 